\title{
Bundled Payments for Ambulatory Surgery Have the Potential to Foster Increased Transparency, Improved Outcomes, Lower Costs, and Increased Capacity for Health Care Systems
}

\author{
Thomas Wilson ${ }^{1}$ and Sohrab Gollogly ${ }^{2}$
}

${ }^{1}$ Global 1, 6125 Paseo Del Norte Suite 210, Carlsbad, CA 92011, United States

${ }^{2}$ Monterey Spine and Joint, 12 Upper Ragsdale, Suite A Monterey, CA 93940, United States

"Corresponding author: Sohrab Gollogly, Monterey Spine and Joint, 12 Upper Ragsdale, Suite A Monterey, CA 93940, United States, Tel: 018312779172; E-mail: sohrab.gollogly@gmail.com

Received date: Nov 28, 2017; Accepted date: Dec 05, 2017; Published date: Dec 12, 2017

Copyright: ( 2017 Wilson T, et al. This is an open-access article distributed under the terms of the Creative Commons Attribution License, which permits unrestricted use, distribution, and reproduction in any medium, provided the original author and source are credited.

\begin{abstract}
Bundled payments are a new payment methodology that is becoming increasingly popular in the United States. Bundled payments are designed to combine all of the costs associated with the delivery of surgical services, including the professional fees of the surgeon, assistant(s), anesthesiologists, and the facility fees of the hospital or ambulatory surgery center into a single bill. This method of reimbursing facilities and healthcare professionals has been utilized by the Federal Government and private insurance carriers in the United States and represents a significant departure from traditional fee for service payment systems. Bundled payments have the potential to decrease health care costs, improve transparency, improve outcomes, and increase the capacity of the health care system by creating an open market for surgical services.
\end{abstract}

\section{Introduction}

The problem of healthcare in the United States is universally acknowledged. Put simply, care is inefficient - efficiency defined as maximizing positive health outcomes while minimizing costs. Standard markets in other domains offer a range of quality to cost options, while "customers" (that is you, us) of the health care system are unable to make efficient choices because we do not have sufficient information to compare one provider to another, or one health care delivery system against another. In essence, we are forced to be inefficient because there is no transparency in the cost to benefit ratio of our individual decisions or the decisions made on our behalf by a health care team.

As a result, we are complicit with spending over $18 \%$ of gross domestic product (GDP) on healthcare, and the rate is only projected to increase [1]. Estimates show health care spending growing 1.3 percentage points faster than GDP during the next decade, reaching $20 \%$ by $2025[2]$.

As we focus on the complexities in healthcare, salient factors include:

- We are faced with a mutually exclusive dichotomy: either healthcare is financially rarefied and inaccessible to a large segment of the population, or it is steeply subsidized, at the risk of fundamentally compromising a host of public services, from education to much-needed investment in urban infrastructure. This subsidization currently consumes $25 \%$ of the U.S. Federal Government Budget and is rising [3].

- Specifically, the United States healthcare system is evolving into a patchwork of, at worst, regional monopolies and, at best, oligopolies. Contained insurance/provider networks are not subjected to market forces that regulate and incentivize improvement. The consumer lacks information, and makes decisions with the mentality of spending someone else's money which collectively amounts to an increase of systemic weight.

- Under the traditional Fee for Service (FFS) model, providers are compensated for the sheer quantity of medical services provided rather than delivering quality outcomes. Most healthcare delivered in the US is billed in isolation, resulting in a system that fundamentally rewards overtreatment instead of patient wellbeing. More patients, more procedures, and more bills create a quantitynot quality-driven balance sheet.

- Payers, including the federal government, commercial insurances, and self-insured employers bring only marginal value to their clients under the current healthcare payment system. Duplication, poor outcomes and even inaccurate diagnoses and treatment, are rewarded, while the systemic outcomes of an open market system innovation, transparency and choice - are being obviated.

- We are sinking an enormous percentage of our visible cost into systemic inefficiency, resulting in an unsustainable burden on the American economy. There is an opportunity to invest in transparency, innovation, choice and affordability universally touted by healthcare policy makers and economists as essential elements of a successful market system, a vital objective at a moment of national change in US Healthcare policy These changes will focus on the consumer taking more responsibility for their care through modifying deductible limits allowing greater diversity of insurance product options and expansion of Health Savings Accounts, and will result in the consumer having more "skin in the game." This will motivate the patient to purchase healthcare on a value basis. Quality, service and cost could be optimized if health care consumers are empowered with the tools to make their choices more efficient and rational. A transparent open market, replete with choices, must be the norm rather than the exception.

The ambition to achieve transparency, innovation, choice and lower cost seems, initially, too lofty a goal. Healthcare is a juggernaut - a 
Citation: Wilson T, Gollogly S (2017) Bundled Payments for Ambulatory Surgery Have the Potential to Foster Increased Transparency, Improved Outcomes, Lower Costs, and Increased Capacity for Health Care Systems. Health Econ Outcome Res 3: S1-105. doi: 10.4172/2471-268X.1000.S1-105

Page 2 of 4

legacy system, with massive infrastructure, bureaucratic inertia, and, as a public necessity, no freedom to fail. We propose a new framework for efficiently delivering a broad spectrum of procedures based on 'outpatient bundling', what could be thought of as collections of procedures or "health packages." Effective bundling of a medical condition has become feasible with the advent of patient-level big data and networked, localized markets of outpatient clinics. Bundled cases have been shown to improve care and reduce costs. We propose a care model based on outpatient services and home-based recovery that can be effectively merged with improved outcomes at the individual patient level. Health packages cause providers to re-focus on the fundamental value proposition of healthcare: improving patient health, streamlining service and reducing cost.

\section{Surgical Care Trending Towards Efficiency}

A bleak picture of the American healthcare landscape is punctuated by notable, albeit limited, examples of cost-and-outcome driven efficiency. In a growing trend, $65 \%$ of all U.S. surgeries are now performed on an outpatient basis, owing to technological advances including long-acting local anesthesia and minimally invasive surgical techniques [4]. More importantly, nearly half of these surgeries are performed in lower cost Ambulatory Surgery Centers (ASCs) - that is, outside of hospitals [5].

There are demonstrable benefits to keeping these procedures in outpatient clinics: patients recover more quickly, procedure costs are significantly lower and there are fewer associated complications. According to the 2015-2016 California Ambulatory Surgery Association benchmarking study involving over 1,000,000 surgical cases, the self-reported surgical site infection rate is 1 per 2000. This was significantly lower than the infection rate in hospital outpatient surgery departments as reported by the Agency for Healthcare Research and Quality [6].

According to Medicare, it is $44 \%$ less expensive to have a procedure in an Ambulatory Surgery Center then in a hospital. This can be attributed to first-order factors - more specialized practitioners in targeted clinics - as well as a spectrum of second-order factors. For example, cross-contamination happens with surprising frequency in hospitals, as the facilities treat patients who have a broad range of illnesses, some of them contagious. Patients requiring general surgery, including orthopedics, are not ill in the contagious sense, and avoiding hospitals altogether significantly reduces the risk of infection. Finally, post-operative recovery is consistently better (faster, with fewer complications) in the home, as patients are incentivized to be mobile, social, and simply get back to the business of daily life (a non-trivial effect of psychological well-being likely plays an important role) [7].

\section{Systematizing and Broadening New Models}

The challenge is to reconfigure our nation's healthcare system in a way that maximizes the potential of emerging approaches to common procedures. ASCs are demonstrating clear success. That success appears, at first blush, to be limited to procedures that meet specific criteria: those that have undergone technical and procedural advances, can be conducted on an outpatient basis, and are amenable to the luxury of in-home recovery.

Further analysis, however, suggests that it is also due to the underlying architecture of ASCs, which is substantively different from a hospital, in a way that may be replicable. The procedure-chain or package of care, from diagnosis to recovery, is structured as a "bundle," and that foundational unit can drive broader systemic change in healthcare, beyond advances in technology. Packaging and pricing associated procedures have the potential to increase transparency, achieve higher quality of care and minimize cost.

Fueled by the inefficient FFS payment model, Congress passed the Affordable Care Act, creating Accountable Care Organizations (ACOs). ACOs derive their revenues from a preset per member per month payment methodology termed a "capitated payment system." In a top-down model, providers bear risks in maintaining and managing the health of the general population under their jurisdiction. Capitation-based systems are brokered by very large healthcare systems (the amalgamation of hospitals, physician groups and other healthcare providers in a geographic area, as a single large corporation), who are incentivized to steer patients into their umbrellas. Health systems are motivated to increase their population pool, resulting in consolidations and further limiting consumer choice. Mergers of health systems stimulate payers to match the leverage that health systems gain, resulting in a few dominant organizations and monopoly-defined consumer costs. All these actions create unsustainable healthcare costs. Data-driven, bundling is the best model to inject the open market system characteristics into the American healthcare system.

The idea of bundled payments is simple: link together all of the individual medical services associated with a medical condition during an episode of care (generally 60-90 days) through an all-inclusive price. Today, individual procedures (diagnosis and testing, anesthesiology, surgery, facility costs, post-acute care, and physical therapy for example) are scattered and paid for individually. Contrary to realworld open markets in healthcare, monolithic and essentially mandatory ACOs reduce the patient's access to information and freedom of choice in providers for each of these procedures.

Bundled payments group statistically correlated procedures into a fixed price package. Patients pay for value, while provider teams are compensated directly for the care provided, and have the autonomy to collectively maximize the efficiency of the team's care delivery system. The model stipulates that compensation is contingent on achieving overall positive health outcomes for each patient, and must be adequate to do so. Provider teams are financially responsible for complications related to the care episode, but compensation is adjusted for risk (acknowledging those patients with a difficult medical history or confounding factors). Teams of care providers have the financial tools and the autonomy they need to ensure an acceptable outcome for a medical condition being cared for.

Specialized providers are incentivized to support each other toward a positive outcome. Multi-specialty collaboration allows each provider to focus on maximizing their offering while aligning with partners. Financial implications and increased accountability between associated providers also spurs innovation. With the ever-increasing level of tracking, it is possible to integrate with a whole host of services that better tailor services for individuals. This includes a wide spectrum of applications that are emerging today including customized technologies to integrate in-home experience and reduce or replace hospital monitoring environments; empowering friends and family members to be essential care providers, through social networks; digitally controlled systems to administer medications and physical therapy routines. Put simply, bundled payments bring efficiencies into the episode of care and refocus healthcare on effectively treating conditions rather than offering medical procedures (as a one-off service). 
Citation: Wilson T, Gollogly S (2017) Bundled Payments for Ambulatory Surgery Have the Potential to Foster Increased Transparency, Improved Outcomes, Lower Costs, and Increased Capacity for Health Care Systems. Health Econ Outcome Res 3: S1-105.doi: 10.4172/2471-268X.1000.S1-105

\section{Data-Driven \\ Outpatient Procedure Bundle \\ "Health Package"}

Health Packages area fixed payment for a comprehensive set of outpatient surgical services during an episode of care for a specific condition. The surgeon/ASC is at risk as payment is contingent upon good outcomes. Health Packages include the ASC, surgeon, anesthesiologist, medical supplies, testing, imaging, physical therapy, home health services and other medical services provided during the episode of care period.

\section{Health Package Joint Replacement ASC Bundle}

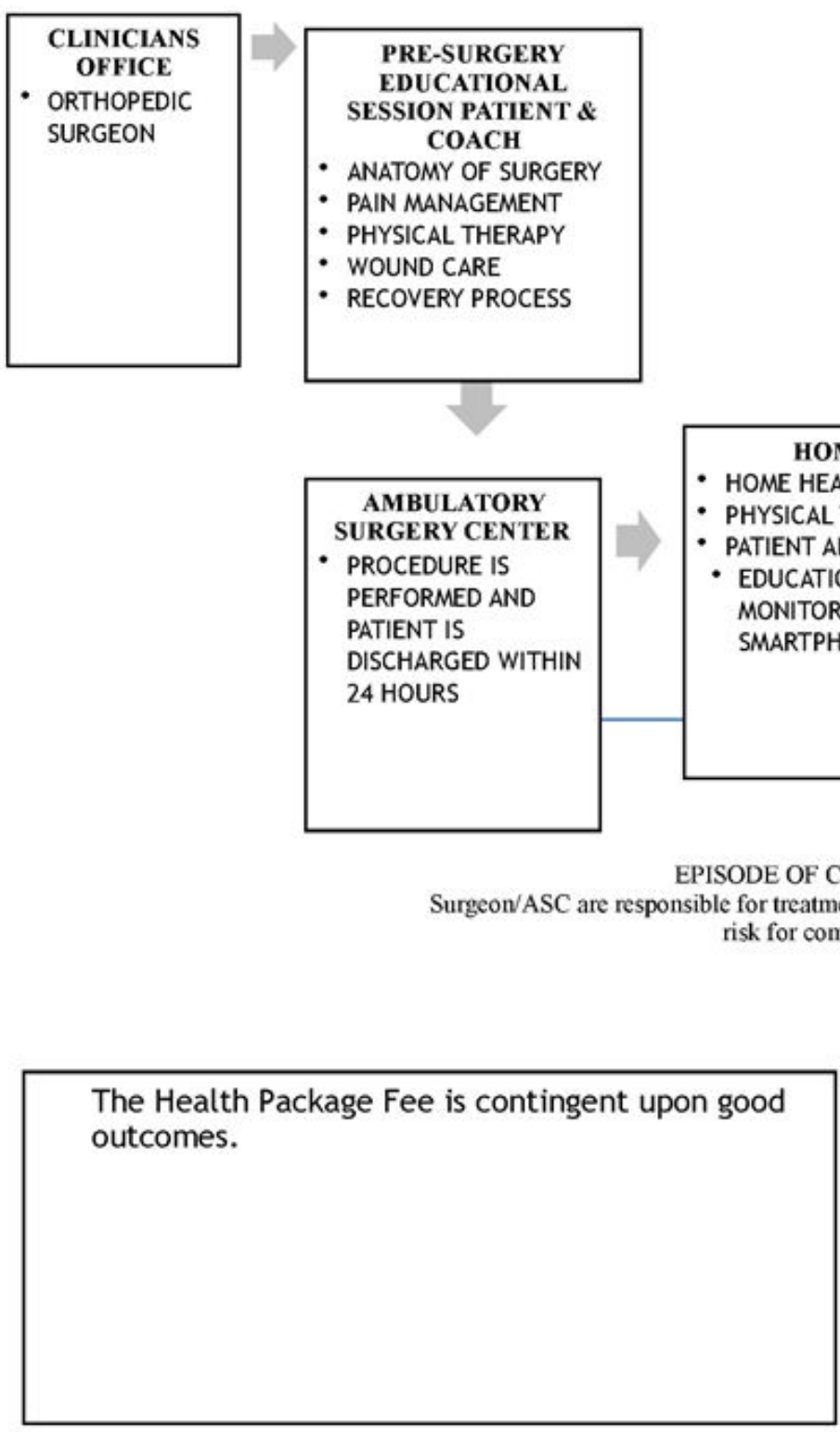

Figure 1: Health package joint replacement ASC bundle.

CLINICIAN OFFICE

- FOLLOW-UP APPOINTMENTS

- ASSESSMENT OF PATIENTS RECOVERY
The Health Package Fee is contingent upon good utcomes.

- PATIENT REPORTED
- PROCEDURE RELATED COMPLICATIONS

- READMISSIONS

- eR VISITS

- infections MEASURES

- patient satisfaction SCORE 
Citation: Wilson T, Gollogly S (2017) Bundled Payments for Ambulatory Surgery Have the Potential to Foster Increased Transparency, Improved Outcomes, Lower Costs, and Increased Capacity for Health Care Systems. Health Econ Outcome Res 3: S1-105.doi: 10.4172/2471-268X.1000.S1-105

Page 4 of 4

Many large healthcare providers support capitation because it maintains the status quo, feeds patients into their existing systems and sharply reduces competition. The top-down approach of capitation enables large healthcare systems to leverage their size and influence. When large incumbents control the capitated dollar, there is a high barrier to entry for competition.

Unlike today's healthcare model, bundled services offer patients freedom to choose a provider team that offers them the package they need. Multiple providers in each region spur competitive innovation (in terms of both procedure and technology adoption) and increase accountability through patient choice - vital factors of controlling costs. Price and outcomes are transparent, subjecting the system to standard market forces. In an era when every product, service and experience is listed and reviewed online, healthcare has been insulated from customer-facing outcome evaluation. Patients should have a simple, understandable view of what they are paying for - shifting from passive to active customers. In an open medical marketplace, provider teams that offer the greatest value - optimizing quality, service and cost - will thrive.

An open medical market requires collection, dissemination and attention to patient satisfaction data. In healthcare, this has traditionally been collected through a costly, cumbersome and lowresolution method, most commonly the Hospital Consumer Assessment of Healthcare Providers and Systems (HCAHPS) survey. Meanwhile, an open market platform is creating high resolution, unbiased data... through the popular review tool Yelp. Recent studies show the correlation between the HCAHPS survey and Yelp is quite strong - for example, high Yelp ratings are correlated with fewer readmissions [8]. Yelp ratings include non-clinical measures such as cost, scheduling, family members care and the compassion of the medical team. These factors are critical to patients and are considered by consumers in making everyday purchase decisions. Open medical markets should acknowledge the user experience of social media and review tools, to provide an improved patient satisfaction system.

\section{Conclusion}

Dramatic changes are happening across many sectors of the US economy as a result of data and technological integration. A prototype of a new payment methodology is emerging in healthcare through Ambulatory Care Centers. These operate on a fundamentally new model that rewards efficiency and value.

We can achieve relevant and measurable human scale outcomes (superior quality, faster recovery, and lower cost) through systematic changes to the American healthcare system, paired with patient-facing advances in technology and process, across the care episode.

Packaged procedures can recalibrate the healthcare system through open market forces. The new framework has the potential to increase competition, hold providers responsible for outcomes, introduce transparency, incentivize innovation and decrease costs. Capitation will do little to reduce health care costs. By its very nature of promoting the status quo, eliminating patient choice, reducing competition and dis-incentivizing innovation, it cannot curb rising healthcare costs. Many leading health care systems are supporting a bundling-based system, with the knowledge that competition and consumer choice will improve overall systemic performance.

Payers such as Blue Shield of California have embraced outpatient bundled payments with great success, providing their clients with better value. Large self-insured groups are beginning to support the Package payment. They are adopting innovative benefit designs that financially incentivize their members to seek centers of value that provide transparent pricing and outcomes through outpatient bundles.

We propose that the route to affordable healthcare - including with open markets, transparency and direct responsibility for outcomes - is through bundled payments. Packaged payments for specific medical conditions are enabled by recent technological developments, and will allow the consumer to purchase healthcare confidently, and to benefit from her investment. A direct, data-driven model can deliver measurable value, individually and systemically.

In healthcare, the Health Package concept creates an open medical market through placing providers at risk for successful treatment of a medical condition. This financial incentive encourages adoption of medical best practices, brings transparency (pricing, quality and service) to the forefront and enables consumers to make an informed decision. It also triggers innovation and competition.

A two-way transfer of data between the patient and the provider will simulate an ongoing inpatient monitoring environment at home, bringing greater efficiency, speed of recovery and transparency to the consumer. Health Packages will unleash competition and expand patient choice as consumers acquire information on prices and complication rates. Patients will have many options and can choose to pursue an outpatient bundle locally or travel to a national center of value to receive care.

Implementation of Health Packages and transparent pricing is now possible through networked platforms which provide in home data analysis to the providers. The smartphone app will recreate the inpatient monitor systems and push information to the patient and their care providers. This will provide essential information to aid the recovery process.

We are at a pivotal juncture in the vitally important healthcare sector. The lack of normal market forces has allowed healthcare to become prohibitively expensive. An open healthcare marketplace will foster sophisticated practices to provide transparency and value in the delivery of medicine, and initiate technological advances to drive down costs and drive efficiencies up. Health Packages are critical to the future of healthcare, creating transparency, consumer choice, innovation, efficiency and savings compared to the current archaic and inefficient system. The future is now, and it is time to act.

\section{References}

1. Centers for Medicare and Medicaid Services (2015) National Health Expenditures 2015 Highlights.

2. Centers for Medicare and Medicaid Services (2015) National Health Expenditure Projections 2015-2025.

3. Congressional Budget Office (2017) The 2017 Long-Term Budget Outlook. pp: 2.

4. Agency for Healthcare Research and Quality (2015) Healthcare Cost and Utilization Project: Statistical Brief \#188.

5. Agency for Healthcare Research and Quality (AHRQ) (2012) Bundled Payment: Effects on Health Care Spending and Quality Closing the Quality Gap: Revisiting the State of the Science (Publication No. 12-E007$\mathrm{EF})$.

6. California Ambulatory Surgery Association (2016) CASA 2016 Benchmarking Report.

7. Meyer, Harris (2016) Replacing joints faster, cheaper and better? Modern Healthcare.

8. Aaron EC (2016) How Yelp Reviews Can Help Improve Patient Care. The New York Times. 